\title{
The Morse Index of a Minimal Surface
}

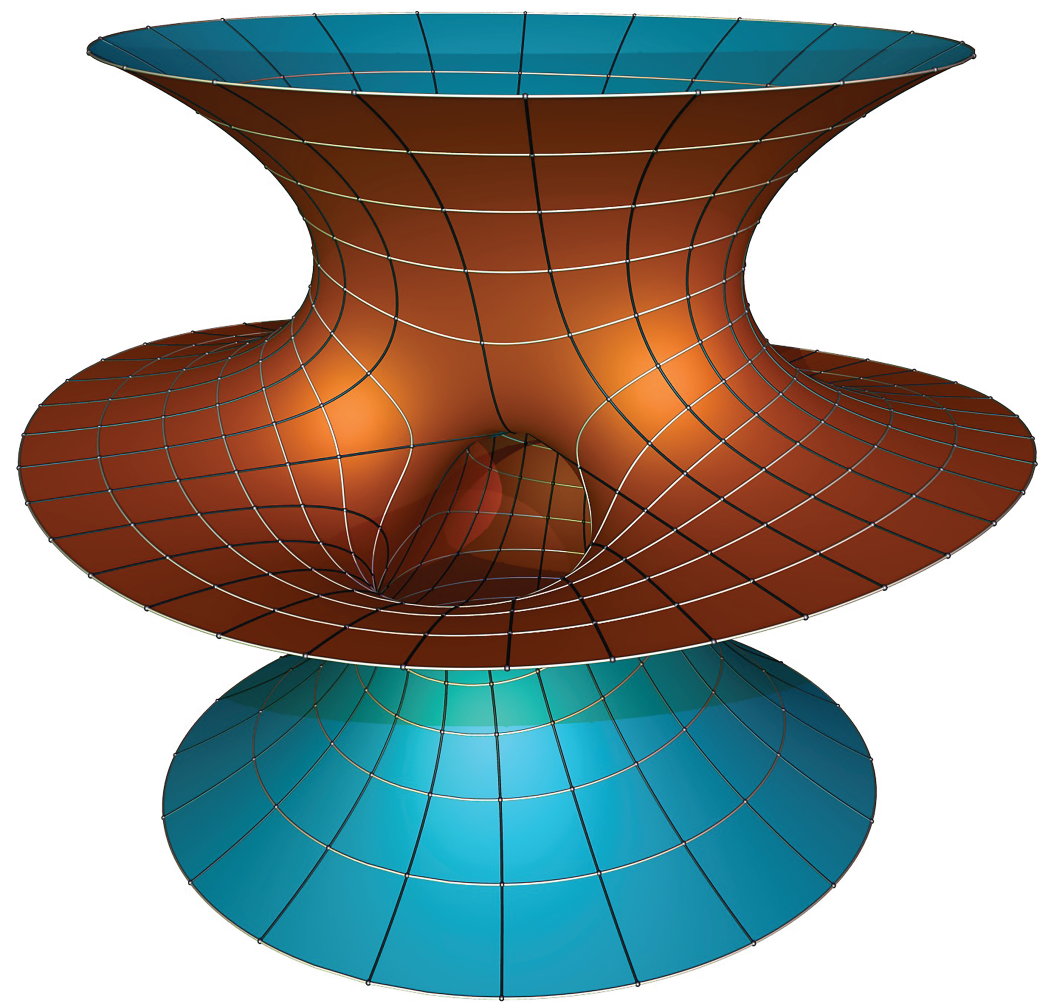

\section{Otis Chodosh and Davi Maximo}

\section{Introduction}

Given a closed curve $\gamma \subset \mathbb{R}^{3}$ we say that a surface $\Sigma \subset \mathbb{R}^{3}$ spans the curve $\gamma$ if $\partial \Sigma=\gamma$. A natural question is:

What surface $\Sigma$ has the least surface area out of all surfaces spanning $\gamma$ ?

We call this Plateau's problem after Joseph Plateau, a 19th century physicist who investigated the behavior of soap films spanning wire contours. However, the question of

Otis Chodosh is an assistant professor of mathematics at Stanford University. His email address is ochodosh@stanford.edu. His research is supported in part by a Terman Fellowship, a Sloan Fellowship, and an NSF grant DMS$1811059 / 2016403$.

Davi Maximo is an assistant professor of mathematics at the University of Pennsylvania. His email address is dmaximamath.upenn.edu. His research is supported in part by a Sloan Fellowship and an NSF grant DMS-1910496.

Communicated by Notices Associate Editor Chikako Mese.

For permission to reprint this article, please contact:

reprint-permission@ams . org.

DOI: https://doi.org/10.1090/noti2291 finding a least area surface spanning a contour dates back at least to work of Lagrange who considered this problem for a graphical surface $z=u(x, y)$ in one of his 1762 foundational works on the calculus of variations.

Lagrange found that the property of having least area implied that the graphical function $u(x, y)$ satisfied a particular partial differential equation (known today as the minimal surface equation):

$$
\left(1+u_{x}^{2}\right) u_{y y}-2 u_{x} u_{y} u_{x y}+\left(1+u_{y}^{2}\right) u_{x x}=0 .
$$

In Figures 1 and 2 we depict the first known examples of minimal surfaces after the flat plane.

Observe that surfaces depicted are not graphs, so what we mean when we say these are minimal surfaces is that each small piece of the surface is graphical over some plane and this graph satisfies the minimal surface equation (1).

\section{The First Variation of Area}

As discovered by Meusnier, the minimal surface equation (1) can be geometrically reformulated as the vanishing of 


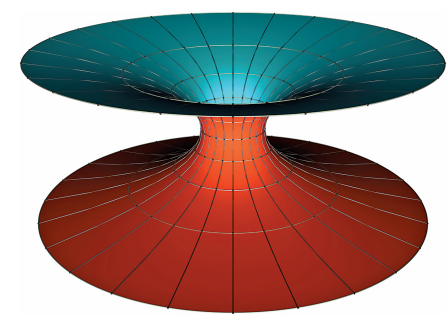

Figure 1. The catenoid (discovered by Euler in 1744 and proven to be minimal by Meusnier in 1776) is the unique axially symmetric minimal surface in $\mathbb{R}^{3}$.

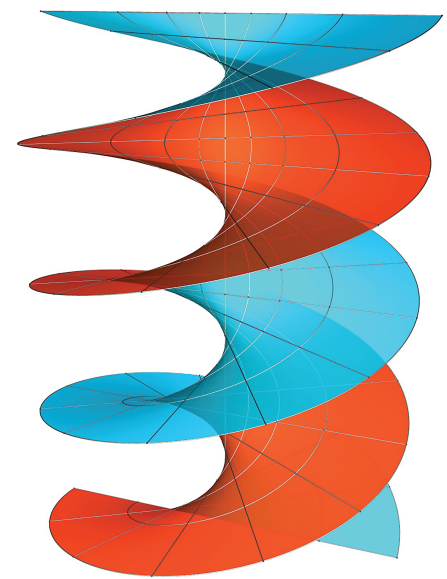

Figure 2. The helicoid (discovered by Meusnier in 1776) is the unique ruled surface in $\mathbb{R}^{3}$.

the mean curvature of the surface. To define the mean curvature, recall that any surface $\Sigma \subset \mathbb{R}^{3}$ (locally) admits a smoothly varying unit normal vector. From this, we can define the Gauss map

$$
N: \Sigma \rightarrow \mathbb{S}^{2}=\left\{x \in \mathbb{R}^{3}:|x|=1\right\}
$$

by setting $N(p)$ to be the unit normal vector to $\Sigma$ at the point $p$. The mean curvature to $\Sigma$ is the trace ${ }^{1}$ (over the tangent plane $T_{p} \Sigma$ ) of the derivative of $N$, i.e.,

$$
H=-\left.\sum_{i=1}^{2} D_{e_{i}} N\right|_{p} \cdot e_{i},
$$

where $e_{1}, e_{2} \in T_{p} \Sigma \subset \mathbb{R}^{3}$ is any orthonormal basis.

If $\Sigma_{t}$ is a smooth family of surfaces ${ }^{2}$ with $\Sigma_{0}=\Sigma$ and so that $\Sigma_{t}$ has velocity $V$ at $t=0$, the first variation of the area formula reads

$$
\left.\frac{d}{d t}\right|_{t=0}\left|\Sigma_{t}\right|=-\int_{\Sigma} H(N \cdot V)
$$

Thus, we see that the vanishing of the mean curvature is precisely the first order condition to be area-minimizing,

\footnotetext{
${ }^{1}$ Classically, the mean curvature was an average (not just sum) of these derivatives, but most modern references omit the factor of $\frac{1}{2}$ here.

${ }^{2}$ If $\Sigma$ is non-compact, we assume that $\Sigma_{t}$ agrees with $\Sigma$ outside of a compact set.
}

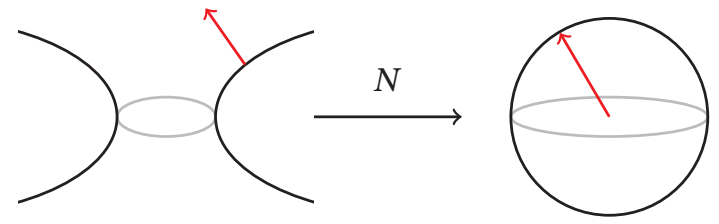

Figure 3. The Gauss map of the catenoid. One can identify the catenoid with $\mathbb{S}^{2} \backslash\{N, S\}$ by "bundling up" both ends. In this case, the Gauss map becomes $N(x)=-x$, an anti-holomorphic map.

since $H$ vanishes if and only if $\left.\frac{d}{d t}\right|_{t=0}\left|\Sigma_{t}\right|=0$ for any family $\Sigma_{t}$.

If $H$ vanishes, then $\Sigma$ is called a minimal surface. Note the potentially confusing nomenclature: a minimal surface need not have minimal area, it is simply a critical point of area. However, a smooth minimal surface is locally of least area in the sense that any point on a minimal surface has a neighborhood that minimizes area.

We note that there are several equivalent properties of minimality. For example, $\Sigma \subset \mathbb{R}^{3}$ is minimal $(H=0)$ if and only if $\Delta_{\Sigma} x^{i}=0$ for $i=1,2,3$, where $x^{i}$ are the coordinate functions on $\mathbb{R}^{3}$. By the maximum principle, this implies that no closed surface on $\mathbb{R}^{3}$ can be minimal. Thus, minimal surfaces in $\mathbb{R}^{3}$ either have boundary, such as solutions of the Plateau problem, or are complete and non-compact.

For us, the most important equivalent characterization will be the following: $\Sigma \subset \mathbb{R}^{3}$ is minimal if and only if the Gauss map $N: \Sigma \rightarrow \mathbb{S}^{2}$ is anti-holomorphic. For example, see the Gauss map of the catenoid in Figure 3.

These facts are the basis of the Weierstrass representation. Loosely speaking, the Weierstrass representation is based on the fact that the Gauss map (an antiholomorphic map on $\Sigma$ ) and $d x_{3}$ (a harmonic 1-form) can be used to recover the minimal surface $\Sigma$. This relates the study of minimal surfaces in $\mathbb{R}^{3}$ to Riemann surface theory, and proves to be a fruitful approach to study existence and uniqueness questions for such surfaces.

\section{The Second Variation of Area}

Returning to the variational approach, if minimal surfaces are critical points of the area functional, it is natural to study the second derivative of area. This is relevant for several reasons. First of all, minimal surfaces are often constructed via variational methods and so the second derivative of area (i.e., the Hessian) plays a central role in the Morse theoretic point of view. Secondly, the second variation is a fundamental geometric object associated to the minimal surface, as we now see.

Suppose that $\Sigma$ is a minimal surface and $\Sigma_{t}$ is a family as before. Modulo a reparametrization of $\Sigma_{t}$, we can assume that the velocity at $t=0$ satisfies $V=\varphi N$ for some function $\varphi$ along $\Sigma$. Then (at least for compactly supported $\varphi$ ) we 


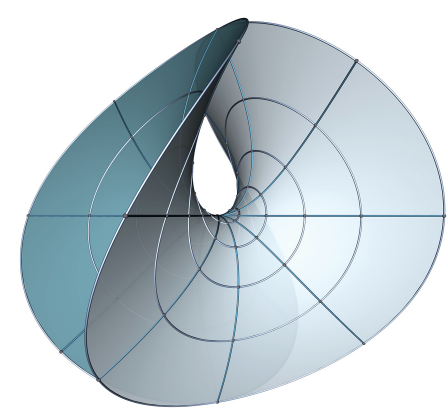

Figure 4. Enneper's surface is an immersed minimal surface in $\mathbb{R}^{3}$. Although it is not obvious from the picture, the induced metric is (intrinsically) rotationally symmetric. Enneper's surface has Morse index 1.

can compute that

$$
\left.\frac{d^{2}}{d t^{2}}\right|_{t=0}\left|\Sigma_{t}\right|=\int_{\Sigma}\left|\nabla_{\Sigma} \varphi\right|^{2}-\left|\nabla_{\Sigma} N\right|^{2} \varphi^{2}
$$

It is common to denote bilinear form on the right hand side of this equation by $Q_{\Sigma}(\varphi)$. This formulation of the second variation of area is due to Fischer-Colbrie [FC85]. She observed that in this form, the second variation $Q_{\Sigma}(\varphi)$ is conformally invariant!

In particular, the second variation $Q_{\Sigma}(\varphi)$ depends only on (1) the conformal class of $\Sigma$ and (2) the Gauss map. Note that (1) and (2) do not necessarily uniquely determine the minimal surface! For example, the catenoid (Figure 1) and Enneper's surface (Figure 4) have (essentially) the same conformal type and Gauss map, although they are clearly not the same minimal surface.

The Morse index. Recalling that $Q_{\Sigma}(\varphi)$ is the second derivative of area in the direction $\varphi$ (really in the direction $\varphi N)$, Morse theory suggests we should study the number of negative and positive eigenvalues of $Q_{\Sigma}(\varphi)$ (thought of as a bilinear form). This is precisely the question of the Morse index of the critical point of area $\Sigma$. It turns out that $Q_{\Sigma}(\varphi)$ always has an infinite number of positive eigenvalues, but may or may not have a finite number of negative eigenvalues.

If $\Sigma$ has a finite number of negative eigenvalues, we say that $\Sigma$ has finite index, and call the number of such eigenvalues the (Morse) index of $\Sigma$ (denoted index $(\Sigma)$ ).

Not all minimal surfaces in $\mathbb{R}^{3}$ have finite index. For example, any periodic minimal surface (other than the plane) has infinite index (for example, the helicoid has infinite index). To see that this is plausible, note that if $\varphi$ has compact support and $Q_{\Sigma}(\varphi)<0$, then translating $\varphi$ by a large number of the periods of symmetries produces a second function $\varphi^{\prime}$ with disjoint support and $Q_{\Sigma}\left(\varphi^{\prime}\right)<0$. This can be repeated and the existence of such functions implies that $\Sigma$ has infinite index.

In fact, as proven by Fischer-Colbrie [FC85], a complete non-compact $\Sigma$ having finite index places strong constraints on the geometry of $\Sigma$. She showed that index $(\Sigma)<\infty$ is equivalent to $\Sigma$ having finite total curvature (FTC), i.e.,

$$
\int_{\Sigma}|K|<\infty
$$

where $K=-\frac{1}{2}\left|\nabla_{\Sigma} N\right|^{2}$ is the Gaussian curvature. The class of finite total curvature surfaces had been studied earlier by Osserman [Oss64] who proved that a finite total curvature minimal surface $\Sigma \subset \mathbb{R}^{3}$ is conformally equivalent to a punctured compact Riemann surface $\bar{\Sigma} \backslash\left\{p_{1}, \ldots, p_{r}\right\}$, and the Gauss map extends anti-holomorphically across the punctures. The reader can compare this to the example of the catenoid discussed previously to see that it is a punctured sphere and the Gauss map extends across the punctures to $\bar{N}: \mathbb{S}^{2} \rightarrow \mathbb{S}^{2}, \bar{N}(x)=-x$.

What's more, Fischer-Colbrie showed that the Morse index of $\Sigma$ (if we know that index $(\Sigma)<\infty$ ) is purely an invariant of the conformal type of $\bar{\Sigma}$ and the extended Gauss $\operatorname{map} \bar{N}$. (This is a consequence of the conformal invariance of $Q_{\Sigma}$, combined with a log-cutoff ${ }^{3}$ argument.)

\section{The Index of the Plane/Catenoid}

We note that for the flat plane, $N$ is constant, so

$$
\mathcal{Q}_{\text {plane }}(\varphi)=\int_{\mathbb{R}^{2}}|\nabla \varphi|^{2} \text {. }
$$

This is always non-negative, so we see that the plane has index (plane $)=0$.

Similarly, for the catenoid, using $\bar{N}(x)=-x$, we see that $\nabla \bar{N}=-\mathrm{Id} \Rightarrow|\nabla \bar{N}|^{2}=2$, so

$$
Q_{\text {catenoid }}(\varphi)=\int_{\mathbb{S}^{2}}\left|\nabla_{\mathbb{S}^{2}} \varphi\right|^{2}-2 \varphi^{2}
$$

Recalling that the first eigenvalue of the Laplace-Beltrami operator on $\mathbb{S}^{2}$ is 0 and the second is 2 , we thus see that index $($ catenoid $)=1$.

\section{Finite Total Curvature Minimal Surfaces}

The set of finite total curvature minimal surfaces (equivalent to finite Morse index surfaces) turns out to be very rich. The class of FTC minimal surfaces is sufficiently restrictive to allow interesting classification results, while being general enough to have a rich set of examples. In large part, this is due to the fact that Weierstrass representation (as mentioned above) allows one to lean on complex analysis when seeking examples of FTC surfaces. Besides the plane, the catenoid (Figure 1), and Enneper's surface (Figure 4), there are many other examples. For example, an immersed example is found in Figure 5.

\footnotetext{
${ }^{3}$ The log-cutoff function uses the Green's function on $\mathbb{R}^{2}$ to produce a sequence of functions $\varphi_{j} \in C_{c}^{\infty}\left(\mathbb{R}^{2}\right)$ with $\varphi_{j} \rightarrow 1$ pointwise but $\int_{\mathbb{R}^{2}}\left|\nabla \varphi_{j}\right|^{2} \rightarrow 0$. This can be used to show that point singularities are "removable" in certain problems.
} 


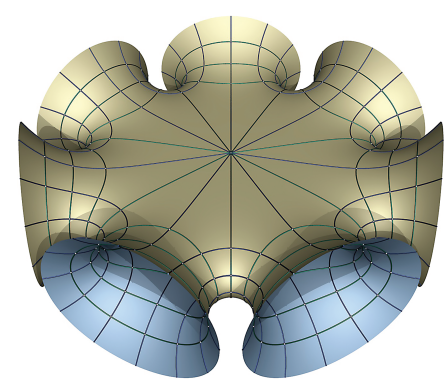

Figure 5. The Jorge-Meeks surfaces are immersed FTC minimal surfaces with $r \geq 3$ catenoidal ends arranged around a circle. The Jorge-Meeks surface with $r$ ends has index $2 r-3$.

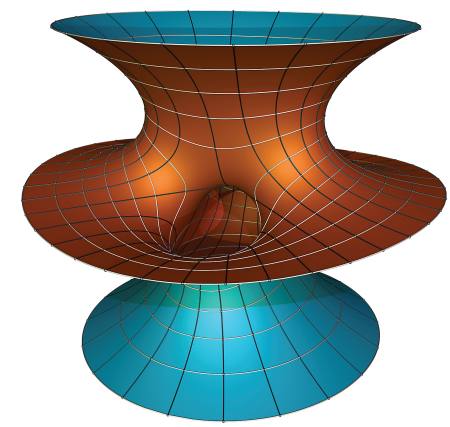

Figure 6. Costa's surface was the first embedded FTC minimal surface (other than the plane and catenoid) to be discovered. The Costa surface has index 5.

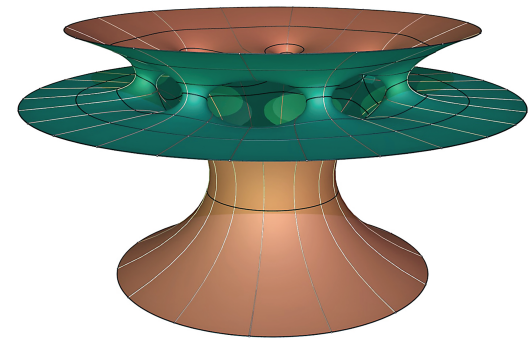

Figure 7. Hoffman-Meeks discovered how to add more handles and bend the middle end of the Costa surface.

A famous and important example of a finite total curvature surface was discovered by Costa in the 1980s [Cos84] (see Figure 6). Costa discovered his surface (conformally a thrice punctured torus) by providing Weierstrass data, but was unable to prove that it was embedded. This was accomplished by Hoffman-Meeks [HM85] who discovered that the surface had certain symmetries and used these to prove embeddedness. They also found generalizations of the Costa surface, in which the middle end bends into a catenoidal shape, and also examples with higher genus. See Figure 7.

The geometry of Costa's surface is considerably more complicated than that of the catenoid. As such, the index of the Costa surface is rather difficult to calculate. This was achieved by Nayatani [Nay93] who showed that index $($ Costa $)=5$ by intricate methods relating the geometry of the second variation operator to the geometry of the Gauss map.

\section{Classification Results}

The class of finite total curvature surfaces is rigid enough to hope to classify simple surfaces. Such results mostly take one of two forms: classification of surfaces of simple topology or low Morse index. These results are rare enough that we can list essentially all of them here.

Classification based on topology. The following results hold for embedded finite total curvature minimal surfaces in $\mathbb{R}^{3}$ :

- The plane is the unique surface with one end.

- The catenoid is the unique surface with two ends (Schoen [Sch83]).

- The plane and catenoid are the unique genus zero surfaces (López-Ros [LR91]).

- The Costa surface (and the deformed version as constructed by Hoffman-Meeks) are the unique genus one surfaces with three ends (Costa [Cos91]).

It would be very interesting to classify embedded genus one FTC minimal surfaces with more than three ends. According to a famous conjecture of Hoffman-Meeks, such surfaces should not exist. More generally they conjecture that

$$
\text { genus } \geq \text { ends }-2 .
$$

Classification based on index. The following results hold for immersed finite total curvature minimal surfaces in $\mathbb{R}^{3}$ :

- The plane is the unique index 0 (stable) surface (Fischer-Colbrie-Schoen [FCS80], do CarmoPeng [dCP79], Pogorelov [Pog81]). ${ }^{4}$

- There is no stable one-sided surface (Ros [Ros06]).

- There is no index 1 one-sided surface (the authors [CM18]).

- The catenoid and Enneper's surface are the unique index 1 surfaces (López-Ros [LR89]).

- There are no index 2 surfaces (the authors [CM16, CM18]).

- There are no index 3 embedded surfaces (the authors [CM18]).

Based on the known examples, it is tempting to conjecture that if $\Sigma \subset \mathbb{R}^{3}$ is an embedded minimal surface with index $(\Sigma) \leq 5$, then $\Sigma$ is a plane, catenoid, Costa's surface, or a member of the Hoffman-Meeks deformation of the latter. Many aspects of this conjecture remain unresolved; in particular it is not known what the precise index

${ }^{4}$ We remark that this classification result and higher dimensional analogues lead to fundamentally important curvature estimates for stable minimal hypersurfaces in Riemannian manifolds. 
of the Hoffman-Meeks deformation family is, much less that there is no other surface with index $(\Sigma) \leq 5$.

\section{Relating Index and Topology}

Recall that Fischer-Colbrie proved that a minimal surface $\Sigma$ in $\mathbb{R}^{3}$ has index $(\Sigma)<\infty$ if and only if $\Sigma$ has finite total curvature. Using the Gauss-Bonnet formula and Osserman's compactification theorem, one can prove the following Jorge-Meeks formula quantizing the total curvature of $\Sigma$ in terms of the topology ${ }^{5}$ of $\Sigma$ :

$$
\int_{\Sigma}|K|=4 \pi \text { (genus }+ \text { ends }-1 \text { ). }
$$

Thus, based on Fischer-Colbrie's "finite index $\Leftrightarrow$ finite total curvature" result, it is natural to ask if there is such a formula for the Morse index.

Based on the examples of surfaces with explicitly computed index, it seems unlikely that such an exact formula would hold. However, it turns out that the index and total curvature are linearly related:

$$
c_{1}+c_{2} \int_{\Sigma}|K| \leq \operatorname{index}(\Sigma) \leq C_{1}+C_{2} \int_{\Sigma}|K| .
$$

The upper bound was proved by Tysk [Tys87], while the lower bound was proved by the authors [CM16, CM18] (one can obtain explicit values for the constants here).

This provides a quantitative version of Fischer-Colbrie's theorem. More importantly, the lower bound for the index (when stated in a more precise form) allows one to prove classification results for low-index surfaces. The precise bound is

$$
\frac{1}{3}(2 \text { genus }+4 \text { ends }-5) \leq \operatorname{index}(\Sigma)
$$

for $\Sigma$ an embedded minimal surface in $\mathbb{R}^{3}$.

Applications to low-index surfaces. We explain how (3) can be used to classify low-index surfaces. For the sake of clarity, we consider here an embedded minimal surface $\Sigma$ with index $(\Sigma)=0,1$ (these cases were already known by previous methods as discussed above).

If index $(\Sigma)=0$, then (3) yields

$$
2 \text { genus }+4 \text { ends } \leq 5 \text {. }
$$

Thus, $\Sigma$ has at most one end, so it must be a plane (by the topological classification results listed above).

If index $(\Sigma)=1$, then (3) yields

$$
\text { genus }+2 \text { ends } \leq 4 \text {. }
$$

We know that $\Sigma$ has at least two ends (since it is not the plane). Thus, it has exactly two ends and is genus zero. Either one of these would suffice to conclude that $\Sigma$ is the catenoid (by the topological classification results listed above).

\footnotetext{
${ }^{5}$ One must modify this equation when $\Sigma$ has non-embedded ends.
}

The non-existence of index $(\Sigma)=2,3$ surfaces follows from similar but more involved arguments.

\section{The Harmonic Form Method}

We briefly comment on the proof of the lower bound (3). There are special methods that can be used to study index 0 or 1 surfaces, but to go beyond this one needs a general way of relating the geometry (the index) to the topology of the surface; this can be accomplished by using harmonic (holomorphic) 1-forms. This approach was pioneered by Ros [Ros06], using some test functions constructed by Palmer [Pal91], and later extended by the authors [CM16, CM18].

Because the second variation operator takes functions and not forms, one must turn the 1-forms into functions. One way to do so, while preserving the vector space structure of the space of harmonic 1-forms, is to take a 1-form $\omega$ on $\Sigma$ and form the inner product $\left\langle\omega, \star d x^{i}\right\rangle$, where $\star$ is the Hodge star operator on $\Sigma$. One reason that this is a fruitful choice is that, as we have seen above, the coordinate functions $x^{i}$ are harmonic on $\Sigma$.

If the topology of $\Sigma$ is large, there are a lot of harmonic 1 -forms. So, if the index is smaller than expected, we can find $\omega$ so that $\left\langle\omega, \star d x^{i}\right\rangle$ is $L^{2}$-orthogonal to the index. Using the Bochner formula, Ros proved that this property is highly constraining (only a linear combination of $\star d x^{i}$, $i=1,2,3$ can satisfy this property, corresponding to an ambient translation of $\Sigma$ ).

It thus remains to find harmonic 1-forms that are allowable in the above argument. Ros used harmonic forms that are in $L^{2}$. Because the $L^{2}$ norm of a 1 -form on a surface is conformally invariant, one can show that the space of $L^{2}$-harmonic 1 -forms on $\Sigma$ is the same as the space of harmonic 1-forms on the compactified $\bar{\Sigma}$, i.e., twice the genus.

The key observation in [CM16, CM18] is that by replacing $L^{2}$ with an appropriately weighted $L^{2}$-space, one can find more harmonic 1 -forms (corresponding to the ends). This improves the estimate to the point where it can be used to classify low-index surfaces.

1 -forms on the catenoid. If one considers the estimate (3) for $\Sigma$ the catenoid, we note that genus $=0$, ends $=2$ so the equation reads

$$
\underbrace{\frac{1}{3}(2 \times 0+4 \times 2-5)}_{=1} \leq \text { index }(\text { catenoid })=1 .
$$

So we can see that (at least in this case) the estimate is sharp. We now explain the various 1 -forms. Recalling that the catenoid is conformally equivalent to $\mathbb{S}^{2} \backslash\{N, S\}$, it is useful to choose a different representative of the conformal class: $\mathbb{C} \backslash\{0\}$ (this allows us to make direct contact with complex analysis). What are the harmonic forms on $\mathbb{C} \backslash\{0\}$ ? We know they are the real/imaginary parts of holomorphic forms. Some simple forms we can think of are 
$d z, \frac{d z}{z}, \frac{d z}{z^{2}}$. It turns out that these forms are precisely the ones that can be used in the weighted spaces described above (forms such as $z d z, \frac{d z}{z^{3}}$ turn out to grow too quickly along the ends). This yields 6 harmonic forms, but we must ignore 3 of them coming from the coordinate functions. This yields 3 forms, and we divide by 3 corresponding to the way we plug them into the second variation; this yields 1 as expected!

\section{The Bigger Picture}

We conclude by discussing how this fits into the broader study of the Morse index and Morse theoretic methods for producing and studying minimal surfaces, as well as point out several open questions.

The index as a geometric invariant. The Morse index is an interesting invariant of a rich class of objects (finite total curvature minimal surfaces) that have been studied since the beginnings of differential geometry. This alone motivates its study. From this point of view, one can ask the following simple question: What is the set

$$
I_{\mathrm{emb}}:=\left\{\operatorname{index}(\Sigma): \Sigma \subset \mathbb{R}^{3} \text { emb. minimal surface }\right\}
$$

of non-negative integers (one can also replace "embedded" with "immersed" and ask the same question)? We have seen that $\{0,1,5\} \subset I_{\text {emb }}$. Moreover, the higher genus Costa surfaces constructed by Hoffman-Meeks turn out to have index 2 genus +3 when the middle end is flat. ${ }^{6}$ Thus, combining these examples with our non-existence results, we can say that

$$
\{0,1, \mathcal{2}, \not, ?, ?, ?, 7, ?, 9, \ldots\} \subset I_{\mathrm{emb}} .
$$

We don't know about any of the even numbers bigger than 2 , although it seems likely that $4 \notin I_{\mathrm{emb}}$. As we have discussed above, it would be very interesting to classify $\Sigma$ with index $(\Sigma) \leq 5$.

There are many more examples of FTC surfaces in $\mathbb{R}^{3}$ that are constructed using various methods (gluing, Weierstrass, etc.). However, we do not know the index of any of them (a wonderful geometric idea of Choe [Cho90] allows us to estimate the index from below, but it does not seem like this gives sharp estimates in general). Just to give a concrete example, we do not know the index of the CostaWohlgemuth surface in Figure 8.

The Morse index in manifolds. As first observed by Schoen and Yau, the existence of stable minimal hypersurfaces of certain topological type poses restrictions on the scalar curvature of the ambient manifold, and viceversa. They used this to prove (among other things) the celebrated positive mass theorem [SY79]. This, along with

\footnotetext{
${ }^{6}$ At present, nobody knows how to compute the index of the surfaces with a bent middle end.
}

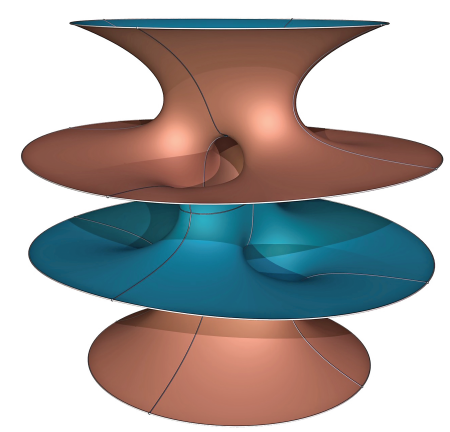

Figure 8. Wohlgemuth generalized the Costa surface by adding an extra flat end. This does not contradict the Hoffman-Meeks conjecture (2), since it has genus two, not one. The index of this surface is not known.

related work, initiated a more systematic study of the relationship between the Morse index and topology of a minimal submanifold as it relates to the geometry of the ambient manifold.

More recently, unstable minimal submanifolds have been used by Marques and Neves on their solution of the Willmore conjecture. Since then there has been an explosion of activity towards the study of Morse theoretic properties of the area functional on submanifolds of Riemannian manifolds. The basic idea here is to find/study minimal surfaces based on the topology of the space of surfaces in the manifold, and the geometry of the area function on this space.

This is an intricate and technical field, but it has had remarkable successes. For example, famous work of Almgren-Pitts (along with important contributions by Schoen-Simon) shows that any Riemannian manifold admits a minimal hypersurface. Thanks to recent work of Marques-Neves and others, this surface will have Morse index at most 1. In fact, these ideas have led to the following result of Song resolving the general case of a well-known conjecture by Yau: any Riemannian manifold $\left(M^{n}, g\right)(3 \leq n \leq 7)$ admits infinitely many embedded minimal hypersurfaces. We recommend the recent survey article by Marques-Neves [MN20] for an excellent exposition of these and more exciting results in this direction.

In some cases (although not all), one knows even more: there is at least one minimal hypersurface of each positive Morse index in certain manifolds. Thus, to study the properties of such minimal hypersurfaces, one might place various restrictions on the index or topology and investigate this class of surfaces. It turns out that studying the manner in which bounded index minimal hypersurfaces can degenerate leads to a rather complete and satisfying theory. In particular, bounded index minimal hypersurfaces degenerate precisely by "bubbling off" bounded index hypersurfaces in $\mathbb{R}^{n}$. So, a refined understanding of such surfaces can lead to refined results about such degeneration. 
For example, our results [CM16, CM18] have recently been applied in this direction by Ambrozio-BuzanoCarlotto-Sharp. In fact, similar work of the same authors suggested that further investigation of our index bounds (3) for surfaces with symmetries could have applications to degeneration of free boundary minimal surfaces; Shuli Chen has recently obtained a result along these lines.

\section{References}

[CM16] Otis Chodosh and Davi Maximo, On the topology and index of minimal surfaces, J. Differential Geom. 104 (2016), no. 3, 399-418, MR3568626

[CM18] Otis Chodosh and Davi Maximo, On the topology and index of minimal surfaces II, arXiv 1808.06572 (2018).

[Cho90] Jaigyoung Choe, Index, vision number and stability of complete minimal surfaces, Arch. Rational Mech. Anal. 109 (1990), no. 3, 195-212, DOI 10.1007/BF00375088. MR1025170

[Cos91] C. J. Costa, Classification of complete minimal surfaces in $\mathbf{R}^{3}$ with total curvature $12 \pi$, Invent. Math. 105 (1991), no. 2, 273-303. MR1115544

[Cos84] Celso J. Costa, Example of a complete minimal immersion in $\mathbf{R}^{3}$ of genus one and three embedded ends, Bol. Soc. Brasil. Mat. 15 (1984), no. 1-2, 47-54, DOI 10.1007/BF02584707. MR794728

[dCP79] M. do Carmo and C. K. Peng, Stable complete minimal surfaces in $\mathbf{R}^{3}$ are planes, Bull. Amer. Math. Soc. (N.S.) $\mathbf{1}$ (1979), no. 6, 903-906, DOI $10.1090 /$ S0273-0979-197914689-5. MR546314

[FC85] D. Fischer-Colbrie, On complete minimal surfaces with finite Morse index in three-manifolds, Invent. Math. 82 (1985), no. 1, 121-132, DOI 10.1007/BF01394782. MR808112

[FCS80] Doris Fischer-Colbrie and Richard Schoen, The structure of complete stable minimal surfaces in 3-manifolds of nonnegative scalar curvature, Comm. Pure Appl. Math. 33 (1980), no. 2, 199-211, DOI 10.1002/cpa.3160330206. MR562550

[HM85] David A. Hoffman and William Meeks III, A complete embedded minimal surface in $\mathbf{R}^{3}$ with genus one and three ends, J. Differential Geom. 21 (1985), no. 1, 109-127. MR806705

[LR89] Francisco J. López and Antonio Ros, Complete minimal surfaces with index one and stable constant mean curvature surfaces, Comment. Math. Helv. 64 (1989), no. 1, 34-43, DOI 10.1007/BF02564662. MR982560

[LR91] Francisco J. López and Antonio Ros, On embedded complete minimal surfaces of genus zero, J. Differential Geom. 33 (1991), no. 1, 293-300. MR1085145

[MN20] Fernando C. Marques and Andre Neves, Applications of min-max methods to geometry, Geometric analysis, 2020, pp. 41-77.

[Nay93] Shin Nayatani, Morse index and Gauss maps of complete minimal surfaces in Euclidean 3-space, Comment. Math. Helv. 68 (1993), no. 4, 511-537, DOI 10.1007/BF02565834, MR1241471
[Oss64] Robert Osserman, Global properties of minimal surfaces in $E^{3}$ and $E^{n}$, Ann. of Math. (2) 80 (1964), 340-364, DOI 10.2307/1970396. MR179701

[Pal91] Bennett Palmer, Index and stability of harmonic Gauss maps, Math. Z. 206 (1991), no. 4, 563-566. MR1100840

[Pog81] A. V. Pogorelov, On the stability of minimal surfaces (Russian), Dokl. Akad. Nauk SSSR 260 (1981), no. 2, 293295. MR630142

[Ros06] Antonio Ros, One-sided complete stable minimal surfaces, J. Differential Geom. 74 (2006), no. 1, 69-92. MR2260928

[SY79] Richard Schoen and Shing Tung Yau, On the proof of the positive mass conjecture in general relativity, Comm. Math. Phys. 65 (1979), no. 1, 45-76. MR526976

[Sch83] Richard M. Schoen, Uniqueness, symmetry, and embeddedness of minimal surfaces, J. Differential Geom. 18 (1983), no. 4, 791-809 (1984). MR730928

[Tys87] Johan Tysk, Eigenvalue estimates with applications to minimal surfaces, Pacific J. Math. 128 (1987), no. 2, 361366. MR888524

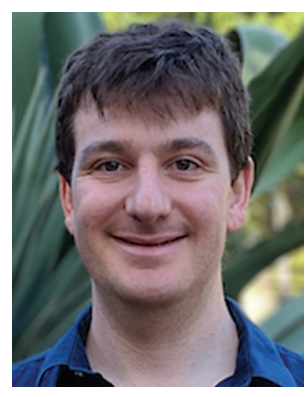

Otis Chodosh

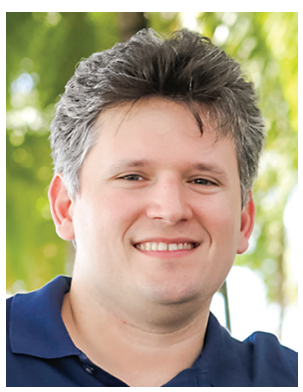

Davi Maximo

\section{Credits}

Opening image and Figures $1-3$ and 5-8 are courtesy of Matthias Weber. Licensed under Creative Commons Attribution Share Alike license 4.0.

Figure 4 is courtesy of Otis Chodosh and Davi Maximo. Photo of Otis Chodosh is courtesy of Otis Chodosh. Photo of Davi Maximo is courtesy of Davi Maximo. 